\title{
Multiscale Detection of Gesture Patterns in Continuous Motion Trajectories
}

\author{
Radu-Daniel Vatavu ${ }^{1}$, Laurent Grisoni ${ }^{2}$, and Stefan-Gheorghe Pentiuc ${ }^{1}$ \\ 1 University Stefan cel Mare of Suceava, Romania \\ 2 Laboratoire d'Informatique Fondamentale de Lille, France \\ vatavu@eed.usv.ro, laurent.grisoni@lifl.fr, pentiuc@eed.usv.ro \\ http://www.eed.usv.ro/ vatavu
}

\begin{abstract}
We describe a numerical method for scale invariant detection of gesture patterns in continuous $2 \mathrm{D}$ motions. The algorithm is fast due to our rejection-based reasoning achieved using a new set of curvaturebased functions. Performance results are reported on a large data set.
\end{abstract}

Key words: gesture recognition, pattern detection, multiscale, curvature, integral of curvature, motion trajectory

\section{Introduction}

Many existing gesture-based interaction systems isolate movements by making use of user-driven discrete events such as mouse clicks, stylus up/down or users being required to maintain a specified posture in vision-based processing. Users are the ones that let the system know when and where gesture commands start and end which has direct impact on the fluidity of the interaction process. The alternative is to automatically detect gestures in constraint-free continuous motions for which we propose a novel technique. The challenge is a difficult one cause of the multiscale issue: given two curves $G(s)$ and $\Gamma(s)$, find the occurences of $G(s)$ in $\Gamma(s)$ regardless of scale.

\section{Curvature-based computations}

The curvature signature function $\kappa(s)$ of a planar curve $C(s)$ parameterized by arc-length $s$ fully prescribes the original curve up to a rigid motion transformation [1] which makes curvature representations suitable for performing gesture recognition as in [2].

We build on top of the elastic matching process of [2] and introduce the principle of summed area tables to the 1D case of the curvature signature function associated to a gesture motion. This way, a new class of monotonic ascending functions are obtained by summing consecutive curvature-based values: $I(s)=\int_{C} f_{+}(\kappa(s)) d s$ where $f_{+}$is a positive function that assures the monotonicity of the integral, e.g. $I_{1}(s)=\int_{C}|\kappa(s)| d s, I_{2}(s)=\int_{C} \kappa^{2}(s) d s$ or $I_{3, \lambda}(s)=\int_{C}|\kappa(s)-\kappa(s-\lambda)| d s$ with $\lambda$ an arc-length constant. Figure 1 illustrates the curvature and integral curvature representations $\left(I_{3, \lambda=1}(s)\right)$ for several executions of a heart gesture. The arc-length $s$ is normalized in the $[0,1]$ interval. 


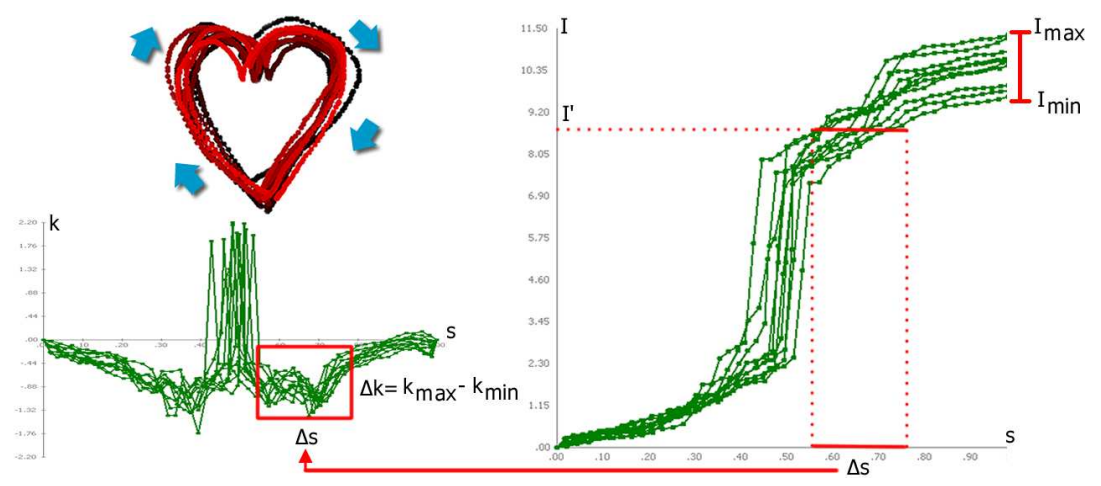

Fig. 1. Curvatures (bottom left) and integral curvatures (right) representations for heart gestures (multiple executions are displayed superimposed).

\section{Multiscale detection algorithm}

Given $p<q$ two indexes on the sampled continuous trajectory $\Gamma(s)$ which define a potential candidate, the integral value for the $\Gamma_{p, q}$ segment is $I_{p, q}=I(q)-I(p)$. The integral function introduces two rules for rejecting candidates as Figure 1 illustrates. First, the interval $\left[I_{\min }, I_{\max }\right]$ is used for scale estimation. Secondly, a given integral value $I^{\prime}$ is associated to a unique arc-length interval $\Delta s$ due to the monotonic ascending property of $I$. Correlating $\Delta s$ with the curvature function, lower and upper margins for curvature at $I^{\prime}$ are obtained: $\Delta k=k_{\max }-k_{\min }$. Parameters $I_{\min }, I_{\max }$ as well as pairs of $\left(I^{\prime}, \Delta s, \Delta k\right)$ for $I^{\prime}$ in $\left[0, I_{\max }\right]$ are computed from training sets for each gesture type. The rules run in $\mathrm{O}(1)$ time. Applying the two rules on $I_{p, q}$ leads to rejection of most candidates.

\section{Results and discussion}

We used a dataset of 1,600 already segmented gesture samples $=16$ types $\mathrm{x} 10$ subjects x 10 executions at normal speed [3]. The 100 samples for each gesture type were equally divided into training and testing. Each gesture from the testing set was inserted with a random scale from $[0.1-0.5]$ in 2 randomly generated trajectories, which gave a testing set of $16 \times 50 \times 2=1,600$ motion trajectories. Detection rate was $94 \%$ with an error of $4.1 \%$ (SD 1.8\%) on the start position and $5.6 \%$ (SD 1.3\%) on the scale. Average execution time was $9.84 \mathrm{~ms}$.

\section{References}

1. Do Carmo, M.: Differential Geometry of Curves and Surfaces. Prentice-Hall (1976)

2. Vatavu, R.D., Grisoni, L., Pentiuc, S.G.: Gesture Recognition based on Elastic Deformation Energies. In: GW'07, Lisbon, Portugal (2007), LNCS 2008.

3. Wobbrock, J.O., Wilson, A.D.,Yang Li: Gestures without libraries, toolkits or training: a $\$ 1$ recognizer for user interface prototypes. In: ACM UIST'07, pp. 159-168. 